\title{
HIGHLIGHTS FROM ISO: THE ISOCAM CAMERA.
}

\author{
CATHERINE J. CESARSKY \\ CEA Saclay/DSM/Service d'Astrophysique \\ Orme des Merisiers, 91191 Gif/Yvette CEDEX
}

\begin{abstract}
Over one year and a half after its first light in orbit, the ISOCAM camera on board the Infrared Space Observatory is bringing back very exciting results. Its spectacular infrared viewing of the Universe sheds new light on the nature of the interstellar dust, on the birth of young stars embedded in dark clouds as well as on bursts of star formation in external galaxies.
\end{abstract}

\section{Isocam}

ISOCAM (Cesarsky et al. 1996) is the camera of the ISO satellite. Its purpose is to provide images of the sky in the 2.5 to $17 \mu \mathrm{m}$ band. It features two array detectors of $32 \times 32$ pixels, one operating in the 2.5 to $5 \mu \mathrm{m}$ range ( $\mathrm{InSb}$ ), and the other in the 4 to $17 \mu \mathrm{m}$ range (Si:Ga).

The camera (Figure 1a) has two independent channels that cannot be used simultaneously: Fabry mirrors, mounted on a selection wheel, deflect the beam towards one array of detectors or the other. The selection wheel has two other positions that enable one of the two arrays to be illuminated with an internal calibration source in order to measure the flat field and to monitor the response of each array throughout the mission. There are two wheels on each channel. The first one carries four interchangeable lenses, allowing to select the spatial resolution. The field of view provided by the ISO telescope is three minutes of arc. At these wavelengths, the spatial resolution is limited by diffraction to a few seconds of arc. The lenses chosen correspond to resolutions of $1.5,3,6$ and 12 seconds of arc per pixel. The second wheel contains ten fixed filters, as well as circular-variable filters with a spectral resolution of 40 . A sixth wheel at the inlet has five positions: two holes and three polarisers, so that polarization measurements are also possible.
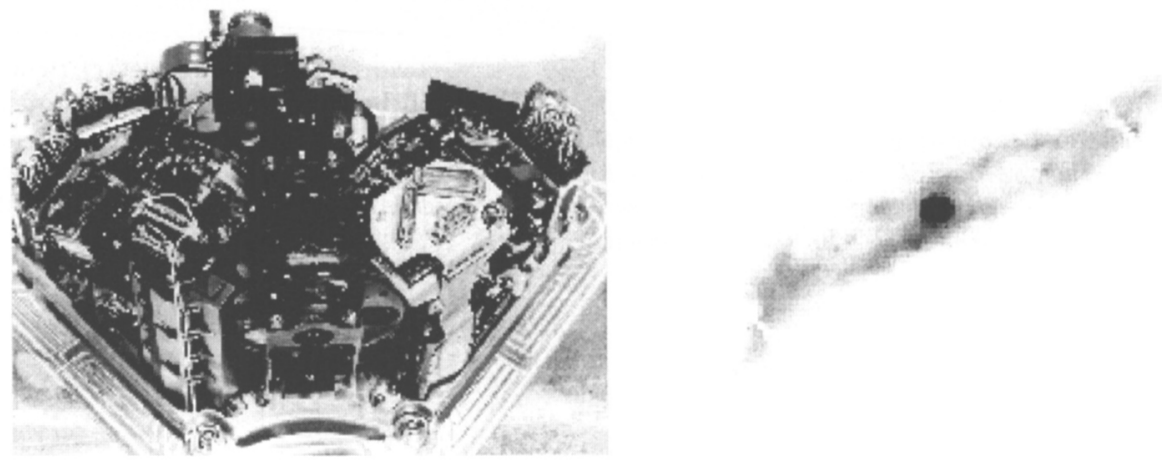

Figure 1. (a) left: A photograph of the focal plane of ISOCAM; (b) right: Centaurus A

The performance of ISOCAM in flight matches or even exceeds the expectations in terms of spatial and spectral resolution, of stability and of sensitivity. The positional accuracy obtained, at 
$15 \mu \mathrm{m}$ and with a pixel field of view of 6 arc seconds, for sources in deep surveys observed on several rasters with displacements of a few pixels, is of 3 arc seconds.

\section{Spectrophotometry of the interstellar medium}

One important aim of the ISOCAM Central Programme is to unravel the various components of the emission of the interstellar medium in the ISOCAM wavelength range: dust and gas heated and excited by UV and/or optical photons, effect of collisional heating, etc. For this, we examined various types of regions and I summarise here the results obtained in some particularly significant cases.

The first important result from ISOCAM has, of course, been the ubiquitous presence of the $6.2,7.7,8.6$ and $11.3 \mu \mathrm{m}$ bands in interstellar and galactic spectra. These bands, which correspond to the stretching and bending modes of $\mathrm{C}-\mathrm{H}$ or $\mathrm{C}-\mathrm{C}$ bonds, had already been observed in planetary or reflexion nebulae. ISOCAM sees them in a very wide range of objects, not only in some bright galactic nebulae and circumstellar envelopes, but also in the cold clouds of our galaxy, the haloes of molecular clouds, the interfaces between HII regions and molecular clouds and also, on a larger scale, in the interstellar medium of spiral galaxies, or in the starburst regions in galaxy mergers. It has even been possible, with the ISOCAM CVF, to draw the spectrum of a cirrus cloud with only one magnitude of extinction (Boulanger et al., in preparation). After subtracting the zodiacal light contribution, previously determined from other ISOCAM observations (Reach et al. 1996), the shape of the spectrum is extremely similar to those in the reflection nebula or HII region interfaces with molecular clouds, even though the intensities of the radiation field in these various environments differ by factors up to $10^{6}$. The fact that these emission spectra are so similar irrespective of the strength of the radiation field indicates that the emission is due to transient heating of hydrocarbon grains.

With ISOCAM, it has also been possible, for the first time, to obtain a direct proof that dust is formed in the "fast moving knots" of the remnant of the Cassiopeia A supernova, known to be made of ejecta from the exploding star (Lagage et al. 1996). Subsequent CVF spectrophotometry has permitted to determine the composition of the gas evaporating from the various knots, thus tracing the history of nucleosynthesis in the star, and to measure the temperature of the dust heated by collisions with the shocked gas (Lagage et al., in preparation).

\section{Star formation in molecular clouds}

In an important programme ISOCAM has been used to map star forming regions in our galaxy. The spectacular two colour ( 7 and $15 \mu \mathrm{m}$ ) map of an area of 0.5 square degree in the Rho Ophiuchi cloud is displayed in Abergel et al. (1996). In this image, the details of the mid infrared emission are revealed: small scale filaments, of thickness about $0.03 \mathrm{pc}$, illuminated by a young, bright star that has emerged from the cloud; limb brightening due to the band features in the cloud halo; presence of dense cores, seen in absorption even at $15 \mu \mathrm{m}$, which probably are stellar progenitors. An intriguing result has been obtained (Olofsson et al. 1997) from a survey of faint objects, at 7 and $15 \mu \mathrm{m}$, in the Chamaeleon I Dark Cloud, complemented by observations in J and $\mathrm{K}$ ' from the DENIS survey (Epchtein et al. 1994). Young stellar objects are easily distinguished by their red colour, indicating the presence of an accretion disk, and a $7 \mu \mathrm{m}$ luminosity function can be established from a sample of 76 objects. For a subsample of these sources, IRAS data are available, so that the bolometric luminosity is well determined; it is surprisingly well correlated to the $7 \mu \mathrm{m}$ luminosity. Assuming that this correlation is still valid at lower luminosities, the $7 \mu \mathrm{m}$ luminosity function can be used to derive the bolometric luminosity function, which in turn can be transformed into a mass function using stellar models and assuming that all the YSOs are roughly coeval with the T Tauri stars of known age in the cloud. The mass function thus obtained extends well into the brown dwarf range of masses, indicating that Chameleon I harbours a population of objects that are good candidates for young brown dwarfs.

\section{Star formation in the Local Group}

ISOCAM also enables us to observe star formation in the neighbouring galaxies. Observations of the large galaxy M 51 at 7 and $15 \mu \mathrm{m}$ (Sauvage et al. 1997) reveal a spiral with two extremely 
fine and regular arms, very different from the more irregular appearance seen in the visible range. The ISOCAM map at $15 \mu \mathrm{m}$ exhibits a closer similarity with a map obtained in the ultraviolet range, and a tight spatial correlation with an $\mathrm{H} \alpha$ map. The ratio of the intensities at $15 \mu \mathrm{m}$ and in $\mathrm{H} \alpha$ varies among HII regions, and is correlated to the visual extinction: thus, maps at $15 \mu \mathrm{m}$, where the radiation is mostly due to reprocessed emission from young ionizing stars, give a more accurate picture of the over all high mass star formation rate in the galaxy than maps in $\mathrm{H} \alpha$ or in the ultraviolet.

This relationship between the $15 \mu \mathrm{m}$ emission and recent star formation is demonstrated even more strikingly in starburst or interacting galaxies. One of the most beautiful examples is provided by the "Antennae" galaxies - a merging system of two spiral galaxies (Vigroux et al. 1996). Analysis of the ISOCAM maps of this object shows that the brightest spot corresponds to a small area, distinct from the two galactic nuclei, and coinciding with the overlap region between the two disks: there, a huge starburst is triggered by the collision. At $15 \mu \mathrm{m}$, this region alone is responsible for about $50 \%$ of the system's emission. CVF maps show that in the emission detected in the $15 \mu \mathrm{m}$ band from this region, the contribution due to ion lines, and in particular to Ne III, is important; the Ne III line traces the region of hardest uv radiation, and its ratio to the Ne II line is well correlated to the over all $15 \mu \mathrm{m}$ emission, indicating again that the emission in this band is a good tracer of high mass star formation.

Another interesting case of merger is provided by the nearby radiogalaxy Centaurus A (Figure 1b). In this case, an elliptical galaxy has collided with a disk galaxy. In the optical, it is essentially the dense dust lanes of the disk galaxy that can be delineated in absorption; the ISOCAM maps reveal the detailed structure of the disk galaxy, whose plane is perpendicular to the radio jets emanating from its nucleus( Mirabel et al. in preparation).

\section{Surveys and galaxy counts}

Surveys at various depths have been performed with ISOCAM, from the very shallow, large area ELAIS survey (led by M. Rowan Robinson) to the shallow, deep and ultra deep surveys in the Central Programme of the ISOCAM team (led by C. Cesarsky), and in other guaranteed and open time projects, including an ultra deep survey in the Hubble Deep Field (Rowan Robinson et al., 1997). Early results of the ISOCAM team deep survey in the Lockman Hole indicate that the slope of the $(\log N-\log S)$ curve at $15 \mu \mathrm{m}$ in the range of one decade around $1 \mathrm{mJy}$, is steep (in excess of 1.7). Strong evolution effects are required to explain this steep slope; this conclusion is reinforced by the counts at the same wavelength in the Hubble Deep Field, yielding results for source intensities above $0.1 \mathrm{mJy}$.

\section{References}

Cesarsky, C.J. et al., A\&A 315, L32, 1996

Reach, W. et al., A\&A 315, L381, 1996

Lagage, P.O. et al., A\&A, L273, 1996

Abergel, A. et al., A\&A, 315, L329, 1996

Olofsson, G. et al., Brown Dwarfs and Extra solar Planets Workshop, Tenerife, 1997, in press

Epchtein, N. et al., Ap and SS, 217, 3, 1994

Sallvage M. et al., A\&A 315, L89,1996

Vigroux L. et al., A\&A 315, L93, 1996

Rowan-Robinson et al., MNRAS, 289, 490, 1997 